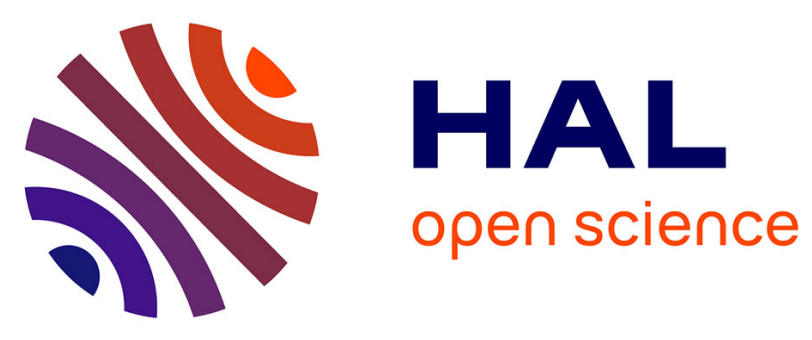

\title{
Pharmacokinetic parameters of infliximab influence the rate of relapse after de-escalation in adults with inflammatory bowel diseases
}

Antoine Petitcollin, Charlène Brochard, Laurent Siproudhis, Camille Tron, Marie-Clémence Verdier, Florian Lemaitre, Camille Lucidarme, Guillaume Bouguen, Éric Bellissant

\section{To cite this version:}

Antoine Petitcollin, Charlène Brochard, Laurent Siproudhis, Camille Tron, Marie-Clémence Verdier, et al.. Pharmacokinetic parameters of infliximab influence the rate of relapse after de-escalation in adults with inflammatory bowel diseases. Clinical Pharmacology and Therapeutics, 2019, 106 (3), pp.605-615. 10.1002/cpt.1429 . hal-02086789

\section{HAL Id: hal-02086789}

\section{https://hal-univ-rennes1.archives-ouvertes.fr/hal-02086789}

Submitted on 8 Jul 2019

HAL is a multi-disciplinary open access archive for the deposit and dissemination of scientific research documents, whether they are published or not. The documents may come from teaching and research institutions in France or abroad, or from public or private research centers.
L'archive ouverte pluridisciplinaire HAL, est destinée au dépôt et à la diffusion de documents scientifiques de niveau recherche, publiés ou non, émanant des établissements d'enseignement et de recherche français ou étrangers, des laboratoires publics ou privés. 


\section{Pharmacokinetic parameters of infliximab influence the rate of relapse after de-escalation in adults with inflammatory bowel diseases}

Antoine Petitcollin ${ }^{1,2,3}$,Charlène Brochard ${ }^{4,5}$, Laurent Siproudhis ${ }^{4,5}$, Camille Tron ${ }^{1,2,3}$, MarieClémence Verdier ${ }^{1,2,3}$, Florian Lemaitre ${ }^{1,2,3}$, Camille Lucidarme ${ }^{5}$, Guillaume Bouguen ${ }^{4,5}$, Éric Bellissant ${ }^{1,2,3}$.

\section{Authors' affiliations}

${ }^{1}$ Rennes University Hospital, Department of Clinical and Biological Pharmacology and Pharmacovigilance, Pharmacoepidemiology and Drug Information Center, Rennes, France.

${ }^{2}$ Rennes 1 University, Faculty of Medicine, Laboratory of Experimental and Clinical Pharmacology, Rennes, France.

${ }^{3}$ Inserm, CIC-P 1414 Clinical Investigation Center, Rennes, France.

${ }^{4}$ Inserm, CIC-P 1414 Clinical Investigation Center, NUMECAN (Nutrition, Metabolism and Cancer) institute, Rennes, France ${ }^{5}$ Rennes University Hospital, Department of Gastroenterology, Rennes, France. 
Corresponding author:

Dr Antoine Petitcollin

Laboratory of Biological Pharmacology

Rennes University Hospital

2 rue Henri Le Guilloux

35033 Rennes

FRANCE

Tel: +33299284280

Fax: +33299284184

Email: antoine.petitcollin@chu-rennes.fr

\section{Keywords}

Infliximab, Inflammatory Bowel Diseases, Population Pharmacokinetics, Therapeutic Drug Monitoring, Dose tapering, De-escalation, Real-World Data

\section{Conflict of Interest}

GB received lecture fees from Abbvie, Ferring, MSD, Takeda and Pfizer and consulting fees from Takeda and Janssen. LS received lecture fees from Abbvie, Ferring and MSD. LS received consultant fees from Takeda and Ferring. AP received lecture fees from Abbvie. All other authors declared no competing interests for this work. 


\section{Funding}

No funding was received for this work.

\section{Abstract}

This study aimed at exploring the link between individual concentrations, pharmacokinetic parameters and the probability of relapse after de-escalation in a real-world prospective cohort of IBD patients who underwent infliximab treatment de-escalation. Ninety-one patients were included. A time-varying compartment model was used to estimate individual pharmacokinetic parameters and trough concentrations. A Cox model was implemented to explore the parameters influencing the probability of relapse after de-escalation. Volume, clearance, and trough before and after de-escalation were linked to the relapse risk at the univariate step. Independent predictors of relapse were tobacco use and/or ulcerative colitis $(p=0.0093)$, a higher C-Reactive Protein $(p=0.00064)$, an infliximab trough $<2.4 \mu \mathrm{g} / \mathrm{mL}$ after de-escalation $(p=0.0001$ ). Patients with trough $>5.7 \mu \mathrm{g} / \mathrm{mL}$ are eligible to de-escalation, but infliximab pharmacokinetics is highly variable in time. Therefore, drug monitoring is mandatory after de-escalation to maintain trough $>2.4 \mu \mathrm{g} / \mathrm{mL}$. Clearance monitoring seem an appealing approach for patient selection and relapse prediction.

\section{Introduction}

Infliximab is a chimeric murine-human monoclonal $\operatorname{lgG} 1 \kappa$ that targets circulating and tissue human Tumour Necrosis Factor $\alpha$ (TNF $\alpha)$. It has proven effective in the management of chronic Inflammatory Bowel Diseases (IBD), namely Crohn's Disease (CD) and Ulcerative 
Colitis $(\mathrm{UC})^{1}$. However, the use of infliximab is associated with an increased risk of cancer, lymphoma and opportunistic infections ${ }^{2,3}$, raising the question of how to reduce these risks in patients with long-term maintenance infliximab treatment. Drug discontinuation has been proposed but led to a high relapse rate ${ }^{4-7}$. Alternatively, treatment de-escalation by lowering the dose or increasing the interval between infusions allows decreasing drug's exposure while maintaining efficacy. Recent data support the relevancy of de-escalation, as higher infliximab levels were reported to be linked to an increased risk of infections ${ }^{8}$. However, data regarding the predictive factors of maintenance of the response after de-escalation of infliximab in IBD are sparse. Amiot and colleagues reported that discrepancies between blind (i.e., based only on clinical data and C-Reactive Protein [CRP]) and therapeutic drug monitoring (TDM)-based dose adjustments led to poorer outcomes after de-escalation ${ }^{9}$. More recently, we demonstrated that TDM-guided selection of the patients before deescalation led to a lower risk of relapse ${ }^{10}$. In this retrospective study, the TDM-based selection of the patients laid on a minimal value of $7 \mu \mathrm{g} / \mathrm{mL}$ before de-escalation, as in the literature ${ }^{11}$ and in accordance with the current knowledge of infliximab pharmacokinetics. However, trough concentrations were not available in all the patients and we were unable to assess the predictive value of the trough concentration on the de-escalation outcomes, neither to determine an optimal concentration threshold of infliximab before and/or after de-escalation associated with a lower relapse rate. Furthermore, no data are available regarding the predictive value of individual pharmacokinetic characteristics on the probability of success of the de-escalation. Identifying these pharmacokinetic characteristics seems an appealing approach, because they are closely linked to the activity of the disease, and recent data suggest that monitoring of infliximab clearance along with the concentrations may be superior to concentrations alone in predicting the success of the 
treatment ${ }^{12}$. Moreover, the long-term pharmacokinetics of infliximab has never been described in IBD patients with sustained remission who underwent a de-escalation, though infliximab pharmacokinetics may be different in this population compared to patients with an active disease, with a direct impact on the modalities of dose adjustments. The use of population pharmacokinetic modelling can help address these questions and refine the exploration of the predictive factors of relapse at de-escalation.

This study aimed at (i) describing the long-term pharmacokinetics of infliximab in a realworld cohort of adults with IBD in deep remission who underwent a de-escalation, and (ii) exploring whether pharmacokinetic parameters and infliximab trough concentrations were associated with successful de-escalation.

\section{Results}

\subsection{Study population}

The study included 91 patients followed up between 2012 and 2017, with a median (IQR) follow-up time of $3.75(2.79-4.82)$ years. Overall, 155 infliximab de-escalation and 44 relapses were recorded. A single de-escalation was performed in 43 patients $(47.3 \%), 36$ patients (39.6\%) experienced 2 de-escalations, and 12 patients (13.1\%) had more than 2 deescalations during their follow-up; 38 patients (40.9\%) experienced 1 relapse, and 4 (4.3\%) experienced more than 1 relapse. The median (IQR) time to relapse (all confounded) was of $280(190-416)$ days after de-escalation. The characteristics of the subjects are summarized in table 1. 


\subsection{Pharmacokinetic analysis}

A total of 607 infliximab concentrations were used to build the pharmacokinetic model. A one-compartment model with time-varying clearance predicted the data well.

The screening of the time-varying covariates revealed that infliximab clearance increased with body weight $(p<0.0001), \operatorname{CRP}(p=0.0017)$, and disease activity $(p=0.00022)$. The influence of the activity was however quantifiable only in UC patients. Azathioprine cotreatment was associated with a slower clearance and was also included as a time-varying covariate in the model $(p=0.0018)$. Overall, 54 increases in infliximab dose were recorded in 44 patients, which were strongly associated with clearance variations (difference in the Objective Function Value $[\Delta \mathrm{OFV}]=-23.1, \mathrm{p}<0.0001)$. In a typical patient, there was a decrease in the clearance of infliximab of $16.9 \%$ when increasing the dose from 5 to $10 \mathrm{mg} / \mathrm{kg}$. All these covariates were retained in the model with an overall $\Delta$ OFV of -93.2 compared to the base model (table 2).

The addition of a linear increase of the clearance with time (denoted to as ) greatly improved the full time-varying covariates model $(\triangle \mathrm{OFV}=-75.4)$, but resulted in the effect of body weight on clearance not being significant anymore, probably indicating a redundancy of and body weight in describing intra-individual clearance variation.

The majority of the remaining concentrations that were not well predicted were properly described after the addition a logit-risk model as previously described ${ }^{12}$, with a decrease of Akaike's Information Criterion (AIC) of -64 . Of note, only 1 sample was tested positive to Anti-Drug Antibodies (ADA) and thus the effect of this covariate was not assessed. 
Eventually, the screening of the static covariates revealed a higher clearance in UC patients $(p=0.00023)$. Because the patients were followed over a long period, some of them had important changes of their corpulence consecutively to disease improvement or worsening. A linear mixed effect model was built to determine the slope of the body weight variations with time over the observation period. The slope of body weight variation partially explained the interindividual variability of the linear increase in clearance (decrease of the unexplained variability of from $29.9 \%$ to $18.8 \%, p=0.0012$ ).

The diagnostic plots showed no obvious model misspecification or bias (figure 1), as well as the Visual Predictive Checks (VPCs) (figure 2). Some typical individual fits are presented in figure 3 , with a representation of the evolution of the clearance with time.

\subsection{Study of the predictive factors of de-escalation outcome}

In the univariate analysis, the probability of relapse after infliximab de-escalation was not influenced by treatment history, demographics or biologics, apart from the CRP which increasing value at the time of the de-escalation increased the risk of subsequent relapse $(p=0.0019)$. Smokers had a higher risk of relapse $(p=0.012)$. The disease type did not influence the probability of relapse, but further investigations revealed that the combined effects of smoking status and disease were linked to the probability of relapse, with a higher risk in smoking CD patients $(p=0.0026)$ and non-smoking UC patients $(p=0.021)$. After gathering the groups with comparable risks, UC patients (whatever the smoking status) and smoking CD patients had a significant higher risk of relapse after de-escalation $(p=0.0031$, figure 4). Conversely, a younger age at diagnosis was slightly associated with a decreased risk of relapse $(p=0.047)$. 
Regarding the pharmacokinetic parameters, a higher volume of distribution was associated with a decreased risk of relapse $(p=0.0075)$, and a higher individual clearance at the time of the de-escalation was associated with poorer outcomes $(p<0.0001)$. Optimal discriminating thresholds were found at $11.3 \mathrm{~L}(p=0.0008)$ and $0.31 \mathrm{~L} / \mathrm{d}(\mathrm{p}=0.0005)$, respectively (figure 4$)$. In total, 2254 trough concentrations were estimated, with a median (IQR) value of 8.0 (4.2 14.9) $\mu \mathrm{g} / \mathrm{mL}$. The probability of relapse increased with infliximab levels at de-escalation $(p=0.014)$, with an optimal discriminating threshold of $<5.7 \mu \mathrm{g} / \mathrm{mL}$ at trough just before deescalation being strongly predictive of the risk of relapse after de-escalation $(p<0.0001$, figure 4). The mean trough concentration calculated from the first trough after de-escalation to the censoring time was also predictive of the risk of relapse of the de-escalation, with an optimal discriminating threshold of $2.4 \mu \mathrm{g} / \mathrm{mL}$ ( $p<0.0001$, figure 4).

After backward selection of the variables, the CRP value at de-escalation, the combined disease/smoking status and the average trough of infliximab after de-escalation were retained as independent predictors of the success of de-escalation (table 3).

\section{Discussion}

This study described the long-term pharmacokinetics of infliximab in adult IBD patients using a time-varying model, emphasizing the wide inter- and intra-individual variability of the clearance over an extended follow-up period. Moreover, this is the first population pharmacokinetic study to focus on treatment de-escalation. Our results show that infliximab concentrations and individual pharmacokinetic parameters are strongly associated with the clinical outcome after de-escalation. 
The values of the estimated pharmacokinetic parameters are in accordance with previous compartment pharmacokinetic studies in adults ${ }^{13-16}$. The data were best described by a onecompartment model with linear elimination. This was expected with a dataset constituted mainly from trough concentrations, which precluded the identification of the distribution process and of the non-linear component of infliximab clearance.

Monoclonal antibodies display a target-mediated elimination. For anti TNF antibodies such as infliximab, this implies that the subjects with an active disease producing high amounts of TNF $\alpha$ have a faster clearance than the patients in remission. Inversely, the blockade of the effects of TNF $\alpha$ by infliximab induces a decrease in the disease-related inflammation, a consecutive decrease in the production of TNF $\alpha$, and ultimately result in a decrease in the clearance of the drug. The fluctuations of the disease activity result, therefore, in variations in infliximab exposure, which are themselves responsible for fluctuations of the disease activity. The influence of several parameters reflecting the inflammation level on the clearance of infliximab has been reported in the literature. In particular, a positive correlation between CRP or disease activity and infliximab clearance has been evidenced ${ }^{14,17}$. Our results are in accordance with this knowledge. However, this influence was characterized using the baseline value of CRP and/or disease activity, and the follow-up of the patients was shorter. In the present study, we have used the clinical scores and the CRP as time-varying covariates. Thus, a CRP of $100 \mathrm{mg} / \mathrm{L}$ increased infliximab clearance by $21.6 \%$. Partial Mayo scores of 3 and 10 resulted in an increase in the clearance of $10 \%$ and $25 \%$, respectively. Conversely, there was no detectable influence of the Harvey Bradshaw Index (HBI) on infliximab clearance, probably because the $\mathrm{HBI}$ correlates poorly with mucosal inflammation ${ }^{18}$. Patients with UC had a $45.8 \%$ faster clearance, in line with a previous pharmacokinetic study ${ }^{19}$. Patients receiving azathioprine co-treatment had a $15.1 \%$ decrease 
in infliximab clearance, which confirms previous reports on the effects of combotherapy ${ }^{20,21}$. This result may be explained by the immunosuppressive effects of azathioprine, which decrease the risk of immunisation to infliximab and the activity of the disease, and consequently the target-mediated elimination of infliximab. Similarly, there was a decrease in infliximab clearance following a dose increase, which was less expected. We hypothesized this was due to the improvement of the disease which led to less target-mediated elimination, rather than because of non-linearity of elimination. Of note, non-linearity was never reported in previous compartment models describing infliximab pharmacokinetics, which comforts our results. These different results emphasize the great influence of the disease activity on infliximab clearance, and they also underline the wide intra-individual variability of infliximab clearance throughout the treatment (figure 3).

Additionally, previous studies have shown that the pharmacokinetics of monoclonal antibodies is time-dependent notably in cancer diseases ${ }^{22-24}$. There is less evidence in inflammatory diseases. It has been shown in adult IBD patients with maintenance infliximab treatment that there was a negative correlation between the number of infusions and infliximab trough concentrations, independently from CRP and clinical scores values, accordingly to our results ${ }^{25}$. In the present study, we modelled a slow linear increase in clearance with time, of $0.0348 \mathrm{~L} / \mathrm{d}$ per year of treatment in a typical subject. Other models such as the sigmoid Emax model, more physiological and used in previous studies, were tested. However, because the shape of the increase in clearance was very close to linear and because this reduced the number of parameters to estimate, a linear model was preferred. This slow increase in clearance was partially correlated to the increase of the body weight. Indeed, in some patients who lost weight, this linear increase was slower but still present. This was never reported before in compartment analysis, but it is important to remain aware 
of this phenomenon, as it may contribute to explain some relapses in patients under longterm maintenance treatment due to a slow evolution towards underexposure to infliximab.

There was a possible, but not systematic, sharp change in clearance in some patients (11/93, 11.8\%). The logit-risk model used here to describe the fast changes in infliximab clearance was already tested in a cohort of children with IBD, and is useful to describe immunisations to infliximab as well as transient periods of higher clearance, for example during the induction phase ${ }^{12}$. Of note, in the present study, only one patient tested positive to ADA. In this patient the logit-risk model detected a clearance increase of 1.9 fold prior to ADA positivity, indicating that the model described the immunization process well in this patient. The rate of immunization in the present cohort also may have been lower than in the general IBD population, because the patients were selected for treatment de-escalation.

Eventually, the implementation of this complex pharmacokinetic model allowed precise estimations of infliximab trough concentrations, which was a pre-requisite for the second part of the study. The survival analysis showed that higher infliximab concentrations were strong predictors of the success of the de-escalation, with a threshold of $5.7 \mu \mathrm{g} / \mathrm{mL}$ before de-escalation and of $2.4 \mu \mathrm{g} / \mathrm{mL}$ at average after de-escalation. Consistently, the median trough concentration at the censure time was $2.2 \mu \mathrm{g} / \mathrm{mL}$ in relapsing patients, compared to $5.5 \mu \mathrm{g} / \mathrm{mL}$ in the others $(\mathrm{p}<0.0001$, Wilcoxon rank-sum test), which confirms the role of the exposure in the maintenance of the response.

We chose to keep into the multivariate analysis only the average concentration after deescalation because the concentrations pre- and post-de-escalation were strongly correlated. This choice is questionable, but we felt that the concentration after de-escalation was more suited as it reflects the actual exposure to infliximab after the de-escalation, whatever the 
concentration before the de-escalation, the individual pharmacokinetic characteristics and/or the modality of the de-escalation (dose, interval or mixed, with several possibilities of dose and/or interval modifications). However, in a typical subject, a 2-weeks increase in the infusion interval or a 2-fold decrease in the dose will approximately result in a 2-fold decrease in the trough after de-escalation, provided there is no disease flare or immunization that would result in increased clearance. Thus, in stable patients, deescalation can be considered if trough infliximab is above $5.7 \mu \mathrm{g} / \mathrm{mL}$.

A higher clearance and a higher linear increase in clearance were logically associated with an increased probability of relapse. These results support our hypothesis that measuring infliximab clearance could be of interest to improve the monitoring of the treatment ${ }^{12}$. A higher volume of distribution was also associated with lower relapse rates. This was unexpected as a higher volume of distribution would result in lower circulating concentrations. The trough concentration values at de-escalation in patients with a volume $\geq 11.4 \mathrm{~L}$ or $<11.4 \mathrm{~L}$ were not different though ( $p=0.08$, Wilcoxon rank-sum test), because of the variability of the dose regimen and of the clearance values. This probably indicates that the effect of volume rather denotes a difference in the distribution process of infliximab. Thus, patients with a higher volume of distribution would have a higher tissue penetration with better efficacy of the drug, which could explain a lower risk of relapse after de-escalation.

The other factors linked with a higher risk of relapse at de-escalation were UC diagnosis, current smoking, and a higher CRP at de-escalation. These last results are in line with the current knowledge of IBD, and are also very close to those reported previously in patients undergoing treatment escalation, were predictors of long-term response were CD diagnosis, non-smoking status and a normal CRP ${ }^{26}$. 
The therapeutic implications of our results are multiple. First, the wide intra-individual variability of infliximab clearance warrants a pro-active TDM, in particular in case of disease flare, to ensure a sufficient exposure. Moreover, the slow increase in clearance we evidenced may lead to underexposure after several years of treatment even in patients in remission. Second, the use of TDM may help to a better selection of the patients eligible to de-escalation. Patients with CD, non-smokers, with low CRP are at lower risk of relapse, but the recourse to TDM increases the probability of success of de-escalation, by selecting only patients that have sufficient concentration before de-escalation (i.e. $>5.7 \mu \mathrm{g} / \mathrm{mL}$ ). Multiple concentrations should be obtained after de-escalation to ensure a sufficient exposure, by maintaining trough concentrations above $2.4 \mu \mathrm{g} / \mathrm{mL}$. Ultimately, the measurement of clearance using compartment modelling may guide dosing adaptations at de-escalation. Indeed, lower increases in the dosing interval are required in "high clearers" to avoid underexposure.

\section{Conclusion}

The results of this study show that the pharmacokinetics of infliximab in IBD adults varies with time. Clearance variations are closely related to the disease activity, the level of inflammation, and the treatment, but there are also unexplained variations such as a slow linear increase that remains only partially explained by body weight variations. Infliximab trough concentrations at de-escalation above $5.7 \mu \mathrm{g} / \mathrm{mL}$ and after de-escalation above 2.4 $\mu \mathrm{g} / \mathrm{mL}$ were associated with better clinical outcomes, as well as a lower clearance and a higher volume of distribution. Non-smoking, a diagnosis of CD and a low CRP at deescalation were also predictive of a better outcome. These results may help to the screening 
of the patients eligible to de-escalation and physicians' decision making, but also emphasize the need of a proactive drug monitoring of infliximab concentrations. A prospective assessment of model-based dosing adaptations to increase the probability of maintenance of the remission is now warranted.

\section{Patients and methods}

\subsection{Patients and study design.}

This was an observational, "real-world", monocentric study, with a retrospective analysis of prospectively-recorded clinical and biological data. The database was approved by the "Commission Nationale Informatique et Libertés" (CNIL \#1412467). The study included 91 IBD adults treated with intravenous infliximab who needed treatment intensification to obtain remission, and who subsequently underwent a treatment de-escalation. The decision of de-escalation was taken by senior physicians experienced in the management of IBD. Patients were eligible to de-escalation if they were in clinical remission, defined as a $\mathrm{HBI}<4$ for $\mathrm{CD}$ or a partial Mayo score $<2$ for UC, and if they were in biological remission, defined as a CRP $<5 \mathrm{mg} / \mathrm{L}$ at the time of the evaluation. De-escalation consisted either of a dose reduction to a minimum of $5 \mathrm{mg} / \mathrm{kg}$ of infliximab, and/or an increase in the interval between infusions of 1 to 4 weeks, to a maximum interval of 12 weeks.

Infliximab trough concentrations were measured before and/or after de-escalation and/or along the treatment period at the discretion of the clinician, using a validated Enzyme-Linked Immunosorbent Assay (ELISA) method ${ }^{27}$. The detection of ADA was performed with an ELISA method previously described ${ }^{28}$. This ADA assay is "drug sensitive", i.e. ADA cannot be 
measured in the presence of infliximab, thus only sera with infliximab level $<0.1 \mu \mathrm{g} / \mathrm{mL}$ were tested for ADA.

Demographics and biologics were recorded at baseline and throughout follow-up at each infusion. The complete history of the treatment was retrieved as well. The clinical response after de-escalation was assessed by the $\mathrm{HBI}^{29}$ or the partial Mayo Score ${ }^{30}$ with respect to the IBD. The success of the de-escalation was defined as the absence of recurrence of clinical and/or endoscopic symptoms, as well as the absence of biological signs of underlying inflammation. Failure was defined as the necessity, based on the aforementioned criteria, to increase infliximab treatment or switch/swap to another treatment. The decision of considering the outcome of a de-escalation as a failure was taken by the physician in charge of the patient, who subsequently adapted the treatment to induce remission of the disease.

\subsection{Pharmacokinetic analysis}

Population pharmacokinetic modelling was performed using Monolix 4.3.3 (Lixsoft; Orsay, France). A one-compartment structural model with zero-order infusion and first-order elimination was implemented and parametrized in terms of clearance $(\mathrm{CL})$ and central volume of distribution (V). Non-linear and mixed (linear and non-linear) eliminations were tested. The pharmacokinetic parameters were assumed to be log-normally distributed:

Where $\theta_{i}$ is the estimated individual parameter for the $\mathrm{i}^{\text {th }}$ patient, $\theta_{T V}$ is the typical value and $\eta_{i}$ is the random effect for the $\mathrm{i}^{\text {th }}$ patient. The values of $\eta_{i}$ were assumed to be normally 
distributed with a mean of 0 and a variance of $\omega^{2}$. For each parameter, was fixed to 0 if the distribution of the $\eta_{i}$ could not be estimated properly.

Additive, proportional and combined additive-proportional residual error models were tested. The combined additive-proportional error model was implemented as follows:

$\mathrm{N}$

where and are observed and predicted $\mathrm{j}^{\text {th }}$ measurements for the $\mathrm{i}^{\text {th }}$ patient, respectively, and and are proportional and additive errors, with a mean of 0 and variances of and , respectively.

\subsection{Investigation of the effect of covariates}

The effect of the following covariates on the pharmacokinetic parameters was investigated: age, gender, body weight, body mass index (BMI), presence of $A D A$, haemoglobin, neutrophil count, lymphocyte count, CRP, disease (CD or UC) and corresponding activity scores ( $\mathrm{HBI}$ and partial Mayo score, respectively), disease phenotype and age at diagnosis according to the Montreal classification, disease duration, infliximab therapy duration, previous treatment by adalimumab, 5-asa and/or glucocorticoids, concomitant use of azathioprine, prior bowel surgery, and tobacco use. Continuous covariates were powertransformed and centred on their median: 
where is the value of for a median subject, quantifies the influence of the covariate on $\theta$, and is the median value of the covariate in the study population.

The influence of categorical covariates was tested with respect to a reference category:

where $\theta_{C A T=0}$ is the value of in an arbitrary reference category and quantifies the influence of the $\mathrm{i}^{\text {th }}$ category on the value of

\subsection{Modelling of the effects of the time-varying covariates}

The poor predictive performance of a simple 1-compartment model with static clearance suggested that infliximab pharmacokinetics varied with time, which was expected given the extended follow-up (median [min - max] of 3.75 [0.93 - 5.34] years). This was attributed to the variations of the activity of the disease and of the level of inflammation, disease flares, body weight variations and/or immunisation to infliximab. Therefore, CRP, body weight, BMI and clinical scores were tested as time-varying covariates. The influence of the co-treatment with azathioprine was tested as well, as a multiplicative factor on the clearance:

Where is the actual clearance of the $i^{\text {th }}$ individual, is the clearance of the $i^{\text {th }}$ individual without azathioprine, is a multiplicative factor that quantifies the influence of azathioprine co-treatment on , and is an indicator variable taking the value 1 if azathioprine is present and 0 otherwise. As a first approach, the same type of equation was used to test the influence of ADA and of treatment intensification, defined as an increase of the dose of infliximab. 


\subsection{Modelling of the time-varying clearance of infliximab}

Despite the use of time-varying covariates, there were still important prediction errors. In particular, there was an overestimation bias that increased with time. As this was already reported before in non-compartment analysis ${ }^{25}$ and suggested by the present data, a timevarying clearance model was implemented that describes a linear increase in clearance with time:

where $\quad$ is the value of infliximab clearance of the $\mathrm{i}^{\text {th }}$ individual at time $t$ (in days), and slo represents the absolute value of the clearance increase over a year.

Nevertheless, there remained some changes in infliximab concentrations that could still not be described with the model above. There were obvious increases in clearance followed by very low concentrations, strongly suggesting the occurrence of an immunization, but without ADA evidenced. Therefore, a logit model was used to detect and describe the clearance variations that were not explained by the aforementioned time-varying model, as we previously reported ${ }^{12}$. Briefly, this model is constituted of a linear risk function of time which is logit-transformed as follows:

where and denote the intercept and the slope of the risk function in the $i^{\text {th }}$ individual, respectively. The logit of the risk, ranging between 0 and 1 , is then applied as a 
power function of a multiplicative term $(C L \quad)$ that quantifies the magnitude of the clearance variation:

$C L \quad$ is assumed to be log-normally distributed and its typical value is assumed to be equal to 1 , meaning that in a typical individual, no change in the clearance is observed.

\subsection{Model comparison and covariate selection}

The objective function was defined as the -2-log-likelihood (-2LL) and the $\Delta \mathrm{OFV}$ was used for model comparison and covariate selection. Nested and non-nested models were compared with the Likelihood Ratio Test (LRT) and AIC, defined as $-2 L L+2 p$ (where $p$ denotes the number of estimated parameters), respectively.

The screening of the relevant time-varying covariates was performed before including the time-varying clearance described above, to avoid rejecting a significant covariate which effect would be inappropriately described by these time-varying functions. The effects of the static covariates were tested after implementing the effects of the time varying covariates and the time-varying clearance. Relevant covariates were selected using the LRT at the univariate step with a risk $\alpha$ of $10 \%$, and were then retained in the full model if their removing resulted in a significant increase of the OFV at a risk $\alpha$ of $1 \%$. The covariate selection was also based on the reduction of the unexplained variability of the parameters, and on the value of the Wald test that verifies whether the values of or are significantly different from 0 . 
The parameters were estimated using the Stochastic Approximation-Expectation Maximization (SAEM) algorithm with simulated annealing. The Relative Standard Errors (RSE) were calculated by stochastic approximation. The model comparison and selection procedures were also based on the inspection of the observed versus individual predicted concentrations, the distribution of the Individual Weighted Residuals, and the inspection of the predictions versus time plot. Prediction-corrected Visual Predictive Checks (VPC) were examined, based on 500 simulated replicates of the dataset. The individual parameters were determined by the Empirical Bayes Estimates (EBE) calculation and were used to estimate the values of the trough concentrations before each infusion.

\subsection{Investigation of the factors influencing the outcome of infliximab de-escalation}

A survival analysis was performed in order to investigate predictive factors of relapse. Because some patients had several consecutive de-escalations, an individual-clustered analysis with repeated events was used. The analysis was performed with Kaplan-Meier curves and univariate comparisons with the Wald test, which does not assume the independence of the observations within a cluster. The numerical variables were tested as continuous covariates and the trough concentration and pharmacokinetic parameters were also transformed into binomial covariates using recursive partitioning if they gave a $p$-value $<0.05$ under their continuous form.

All the variables with univariate tests giving a $p$-value $<0.2$ were included in a multivariate Cox model. The final multivariate model was chosen after backward selection of significant uncorrelated variables. A p-value $<0.05$ was considered as significant. 


\section{Study Highlights}

- What is the current knowledge on this topic?

The pharmacokinetics and concentration-effect relationship of infliximab was never studied in IBD patients undergoing treatment de-escalation. Recent data suggest that the pharmacokinetic parameters, especially the clearance, may be predictive of the clinical response independently from trough concentration.

- What question did this study address?

The pharmacokinetics of infliximab in IBD patients who underwent treatment de-escalation was described using a time-varying clearance model, and the value of trough concentrations and individual clearance in predicting the success of treatment de-escalation was explored.

- What does this study add to our knowledge?

There is a wide intra-individual variability of infliximab pharmacokinetics in IBD patients, partially explained by disease-related parameters. Trough concentration and clearance before de-escalation, IBD type, tobacco use and CRP are strong predictors of relapse after de-escalation.

- How might this change clinical pharmacology or translational science?

The results of this study suggest that monitoring the evolution of the clearance of the patients may help to monitor treatment efficacy and risk of relapse, being a reflection of the disease activity trough target-mediated drug disposition. 


\section{Author Contributions}

A.P., G.B., and E.B. wrote the manuscript; G.B., L.S., and A.P. designed the research; G.B., L.S., C.B., C.L., M.-C.V., F.L., A.P., and C.T. performed the research; A.P. and G.B. analyzed the data; A.P., M.-C.V., F.L., and C.T. contributed new reagents/analytical tools.

\section{Acknowledgements}

The authors wish to sincerely thank all physicians and nurses who were involved in patients' care, as well as the patients themselves. The authors also wish to thank $\mathrm{Pr} \mathrm{H}$. Watier for antidrug antibodies assays, and $\operatorname{Dr}$ C. Lebert for providing data on infliximab treatment supply.

\section{References}

1. Sofia MA, Rubin DT. The Impact of Therapeutic Antibodies on the Management of Digestive Diseases: History, Current Practice, and Future Directions. Dig Dis Sci. 62,833-42 (2017).

2. Williams CJ, Peyrin-Biroulet L, Ford AC. Systematic review with meta-analysis: malignancies with anti-tumour necrosis factor-alpha therapy in inflammatory bowel disease. Aliment Pharmacol Ther. 39,447-58 (2014).

3. Lemaitre $\mathrm{M}$, et al. Association Between Use of Thiopurines or Tumor Necrosis Factor Antagonists Alone or in Combination and Risk of Lymphoma in Patients With Inflammatory Bowel Disease. JAMA. 318,1679-86 (2017).

4. Louis E, et al. Maintenance of remission among patients with Crohn's disease on antimetabolite therapy after infliximab therapy is stopped. Gastroenterology. 142,63-70 e5; quiz e31 (2012).

5. Bortlik $\mathrm{M}$, et al. Discontinuation of anti-tumor necrosis factor therapy in inflammatory bowel disease patients: a prospective observation. Scand J Gastroenterol. 51,196-202 (2016).

6. Reenaers $\mathrm{C}$, et al. Outcomes 7 Years After Infliximab Withdrawal for Patients With Crohn's Disease in Sustained Remission. Clin Gastroenterol Hepatol. 16,234-43 e2 (2018). 
7. Fiorino G, et al. Discontinuation of Infliximab in Patients With Ulcerative Colitis Is Associated With Increased Risk of Relapse: A Multinational Retrospective Cohort Study. Clin Gastroenterol Hepatol. 14,1426-32 e1 (2016).

8. Bejan-Angoulvant $T$, et al. Brief Report: Relationship Between Serum Infliximab Concentrations and Risk of Infections in Patients Treated for Spondyloarthritis. Arthritis Rheumatol. 69,108-13 (2017).

9. Amiot $\mathrm{A}$, et al. Therapeutic drug monitoring is predictive of loss of response after deescalation of infliximab therapy in patients with inflammatory bowel disease in clinical remission. Clin Res Hepatol Gastroenterol. 40,90-8 (2016).

10. Lucidarme $\mathrm{C}$, et al. Predictors of relapse following infliximab de-escalation in patients with inflammatory bowel disease: the value of a strategy based on therapeutic drug monitoring. Aliment Pharmacol Ther. 49,147-54 (2019).

11. Vande Casteele $\mathrm{N}$, et al. Trough concentrations of infliximab guide dosing for patients with inflammatory bowel disease. Gastroenterology. 148,1320-9 e3 (2015).

12. Petitcollin A, et al. Modeling Immunization To Infliximab in Children With Crohn's Disease Using Population Pharmacokinetics: A Pilot Study. Inflamm Bowel Dis. 24,1745-54 (2018).

13. Brandse JF, et al. A Real-life Population Pharmacokinetic Study Reveals Factors Associated with Clearance and Immunogenicity of Infliximab in Inflammatory Bowel Disease. Inflamm Bowel Dis. 23, 650-60 (2017).

14. Buurman DJ, Maurer JM, Keizer RJ, Kosterink JG, Dijkstra G. Population pharmacokinetics of infliximab in patients with inflammatory bowel disease: potential implications for dosing in clinical practice. Aliment Pharmacol Ther. 42,529-39 (2015).

15. Aubourg A, Picon L, Lecomte T, Bejan-Angoulvant T, Paintaud G, Ternant D. A robust estimation of infliximab pharmacokinetic parameters in Crohn's disease. Eur J Clin Pharmacol. 71,1541-2 (2015).

16. Fasanmade AA, et al. Population pharmacokinetic analysis of infliximab in patients with ulcerative colitis. Eur J Clin Pharmacol. 65,1211-28 (2009).

17. Ternant $D$, et al. Pharmacokinetics and concentration-effect relationship of adalimumab in rheumatoid arthritis. Br J Clin Pharmacol. 79,286-97 (2015).

18. Peyrin-Biroulet L, et al. Defining Disease Severity in Inflammatory Bowel Diseases: Current and Future Directions. Clin Gastroenterol Hepatol. 14,348-54 e17 (2016).

19. Passot $\mathrm{C}$, et al. The underlying inflammatory chronic disease influences infliximab pharmacokinetics. MAbs. 8,1407-16 (2016).

20. Ternant $D$, et al. Relationship between inflammation and infliximab pharmacokinetics in rheumatoid arthritis. Br J Clin Pharmacol. 78,118-28 (2014). 
21. Yarur AJ, et al. Concentrations of 6-thioguanine nucleotide correlate with trough levels of infliximab in patients with inflammatory bowel disease on combination therapy. Clin Gastroenterol Hepatol. 13,1118-24 e3 (2015).

22. Li J, et al. Population pharmacokinetics of rituximab in patients with chronic lymphocytic leukemia. J Clin Pharmacol. 52,1918-26 (2012).

23. Gibiansky E, Gibiansky L, Carlile DJ, Jamois C, Buchheit V, Frey N. Population Pharmacokinetics of Obinutuzumab (GA101) in Chronic Lymphocytic Leukemia (CLL) and NonHodgkin's Lymphoma and Exposure-Response in CLL. CPT Pharmacometrics Syst Pharmacol. 3,e144 (2014).

24. Bajaj G, Wang X, Agrawal S, Gupta M, Roy A, Feng Y. Model-Based Population Pharmacokinetic Analysis of Nivolumab in Patients With Solid Tumors. CPT Pharmacometrics Syst Pharmacol. 6,58-66 (2017).

25. Marits $P$, et al. Trough s-infliximab and antibodies towards infliximab in a cohort of 79 IBD patients with maintenance infliximab treatment. J Crohns Colitis. 8,881-9 (2014).

26. Katz $L$, et al. Doubling the infliximab dose versus halving the infusion intervals in Crohn's disease patients with loss of response. Inflamm Bowel Dis. 18,2026-33 (2012).

27. Ternant $D$, et al. An enzyme-linked immunosorbent assay for therapeutic drug monitoring of infliximab. Ther Drug Monit. 28,169-74 (2006).

28. Magdelaine-Beuzelin C, et al. IgG1 heavy chain-coding gene polymorphism (G1m allotypes) and development of antibodies-to-infliximab. Pharmacogenet Genomics. 19,383-7 (2009).

29. Harvey RF, Bradshaw JM. A simple index of Crohn's-disease activity. Lancet. 1,514 (1980).

30. Lewis JD, Chuai S, Nessel L, Lichtenstein GR, Aberra FN, Ellenberg JH. Use of the noninvasive components of the Mayo score to assess clinical response in ulcerative colitis. Inflamm Bowel Dis. 14,1660-6 (2008). 
Table 1. Patients characteristics $(n=91)$

Age at fisrt de-escalation (years)

Male sex

Weight at first de-escalation $(\mathrm{kg})$

$\mathrm{BMI}$ at first de-escalation $\left(\mathrm{kg} / \mathrm{m}^{2}\right)$

Smoking

Current smokers

Ex-smokers

Non-smokers

Age at diagnosis

A1 <16 years

A2 $17-40$ years

A3 Over 40 years

CD phenotype

B1 Non-stricturing, non penetrating

B2 Stricturing

B3 Penetrating

Perianeal disease (additional to B1, B2 or B3)

L1 Ileal

L2 Colonic

L3 Ileocolonic

L4 Upper disease (additional to L1, L2 or L3)

UC phenotype

E1 Ulcerative proctitis

E2 Left-side UC

E3 Extensive

Disease duration at first de-escalation (years)

Duration of infliximab therapy at fisrt de-escalation (years)

Previous treatment by glucocorticoids

Previous treatment by 5 -asa

Previous treatment by immunomodulators

Previous treatment by adalimumab

Previous bowel surgery

De-escalation characteristics

Total number of de-escalations 155

Number of de-escalation per patient

$1(1-2)$

Type of de-escalation

Dose reduction

Infusion interval increase

Mixed (dose and interval)

Combination therapy at de-escalation
$36.9(29.6-46.2)$

39 (42.9)

$66(60-69)$

$23.5(21.2-26.6)$

17 (18.7)

19 (20.9)

55 (60.4)

15 (16.5)

$66(72.5)$

10 (11.0)

$62(68.1)$

43 (69.3)

$12(19.4)$

7 (11.3)

$21(33.9)$

10 (16.1)

18 (29.1)

34 (54.8)

12 (19.4)

29 (31.9)

5 (17.2)

9 (31.0)

15 (51.8)

$9.1(4.1-14.5)$

$2.9(1.5-6.0)$

50 (54.9)

44 (48.4)

57 (62.6)

12 (13.2)

28 (30.8)

Data are expressed as median (first quartile - third quartile) or number (percentage). Disease phenotype is described following the Montreal classification for inflammatory bowel diseases. 
Parameter description

Value

Precision

of the

estimates

Shrinkage

(RSE \%)

(\%)

Fixed effects

$\mathrm{V}(\mathrm{L})$

$\mathrm{CL}_{\text {base }}\left(\right.$ L.day $^{-1}$ )

Volume of distribution

11.5

5

$\beta_{\text {DiseaseUc }}$

$\beta_{\text {CRP }}$

$\beta_{\text {DOSE }}$

$\beta_{\text {Activityuc }}$

factAZA

slope (L.day ${ }^{-1}$.year ${ }^{-1}$ )

Baseline clearance

0.273

0.377

0.0654

$-0.267$

0.0934

0.849

0.0348

31.1

10.9

0.0526

1

Clearance variation magnitude

BSV of the volume of distribution

25.4

44.3

33.3

32.0

102.0

40.4

BSV of the clearance variation magnitude

Additive error magnitude

0.446

20.6

7

29

15

22

30

2

9

31

5

21

fixed
Residual error

$\sigma_{\text {add }}(\mu \mathrm{g} / \mathrm{mL})$

Proportional error magnitude

15
10
19
26
17
11

22
6

49 
Table 3. Factors influencing the rate of relapse after infliximab de-escalation

Variable

Univariate analysis

Multivariate analysis (Cox)

HR $(95 \% \mathrm{Cl}) \quad$ p-value* $\mathrm{HR}(95 \% \mathrm{Cl}) \quad$ p-value*

Demographics and disease history and phenotype

Age

Male sex

Tobacco (non-smokers versus current or former)

Disease (UC versus CD)

Disease and tobbaco

$\mathrm{CD}$, non-smoker (reference)

$C D$, smoker or former

UC, non-smoker

UC, smoker of former

$\begin{array}{lc}1.00(0.98-1.03) & 0.69 \\ 1.40(0.79-2.48) & 0.25 \\ 0.48(0.27-0.85) & \mathbf{0 . 0 1 2} \\ 1.32(0.77-2.29) & 0.31\end{array}$

1

$3.29(1.52-7.15)$

$2.34(1.14-4.82)$

$2.82(1.01-7.85)$

$2.77(1.41-5.44)$

$0.31(0.10-0.99)$

$1.09(0.62-1.92)$

$1.33(0.75-2.40)$

$0.95(0.52-1.75)$

$1.25(0.60-2.57)$

$1.30(0.69-2.45)$
0.0026

0.021

0.048

0.0031

$\mathbf{0 . 0 4 7}$

0.77

0.34

0.88

0.55

0.42
$\mathrm{UC}$ (all) + smokers with CD

Age at diagnosis ( $<16$ years versus $\geq 16$ years)

Previous treatment by glucocorticoids

Previous treatment by 5-asa

Previous treatment by immunomodulators

Previous treatment by adalimumab

Previous bowel surgery

$2.83(1.29-6.23)$

0.0093

Clinical and biological characteristics and treatments at the time of the de-escalation

Disease duration

$1.00(0.96-1.05) \quad 0.83$

$0.98(0.89-1.09) \quad 0.76$

$1.71(0.85-3.42) \quad 0.13$

$1.06(1.02-1.11) \quad \mathbf{0 . 0 0 1 9}$

$0.90(0.69-1.17) \quad 0.42$

$1.00(0.99-1.00) \quad 0.67$

$0.72(0.53-1.01) \quad 0.059$

$1.19(0.59-2.39) \quad 0.62$

$0.81(0.70-0.95) \quad \mathbf{0 . 0 0 7 5}$

$0.33(0.17-0.63) \quad \mathbf{0 . 0 0 0 8 1}$

$38.73(8.11-185)<0.0001$

$5.83(2.16-15.8) \quad 0.00050$

$1.01(1.01-1.03)<0.0001$

$1.16(0.64-2.08) \quad 0.63$

$0.94(0.90-0.99) \quad \mathbf{0 . 0 1 4}$

$0.34(0.21-0.57) \quad<0.0001$

$0.86(0.74-1.00) \quad 0.051$

$0.20(0.12-0.33)<0.0001$

$0.85(0.74-0.98) \quad \mathbf{0 . 0 2 3}$

$0.22(0.13-0.36)<0.0001$
$1.10(1.04-1.17)$

0.00064

$0.27(0.14-0.52)$

0.00010
Average trough after de-escalation $\geq 2.4 \mu \mathrm{g} / \mathrm{mL}$

Infliximab trough at censure time (continuous)

HR: Hazard ratio ; $95 \% \mathrm{Cl}: 95 \%$ confidence interval. * Wald test. ${ }^{\mathrm{a}} \mathrm{HR}$ is given for slope $\mathrm{x} 1000$, to avoid unreadable values of HR and IC95 due to the small values of the slope. 


\section{Figures Legends}

Figure 1. Diagnostic plots. A. Observed versus population-predicted concentrations. B. Observed versus individually-predicted concentrations. C. Individual weighted residuals versus time. D. Distribution of the individual weighted residuals.

Figure 2. Visual Predictive Checks. The grey shaded areas represent the $90 \%$ confidence interval of the predictions at the 5,50 and $95^{\mathrm{e}}$ percentile. The full lines represents the 5, 50 and 95e percentile of the observations. Open circles are the observed concentrations.

Figure 3. Representative individual fits. The full line represents the individually-predicted concentration versus time. Open circles are the observed concentrations. The dashed line represents the value of the clearance versus time (the values of the clearance are reported on the right-handed axis). The patient on the upper-right was tested positive to ADA at time 455.

Figure 4. Kaplan Meyer curves for probability of sustained remission after de-escalation. 


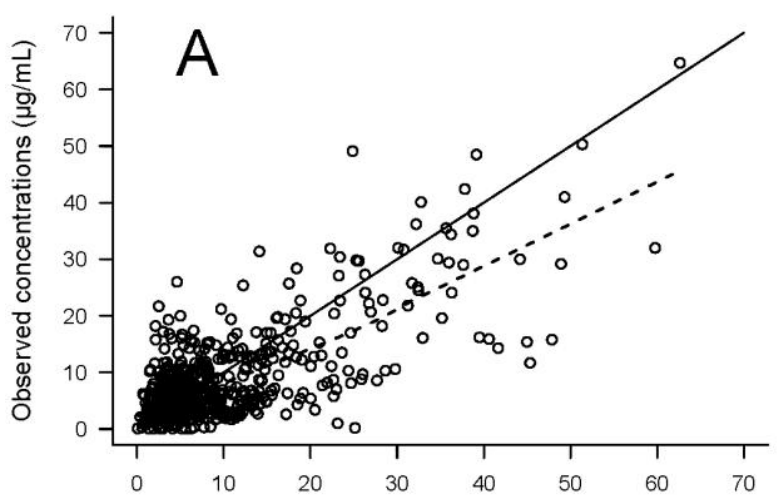

Population-predicted concentrations $(\mu \mathrm{g} / \mathrm{mL})$
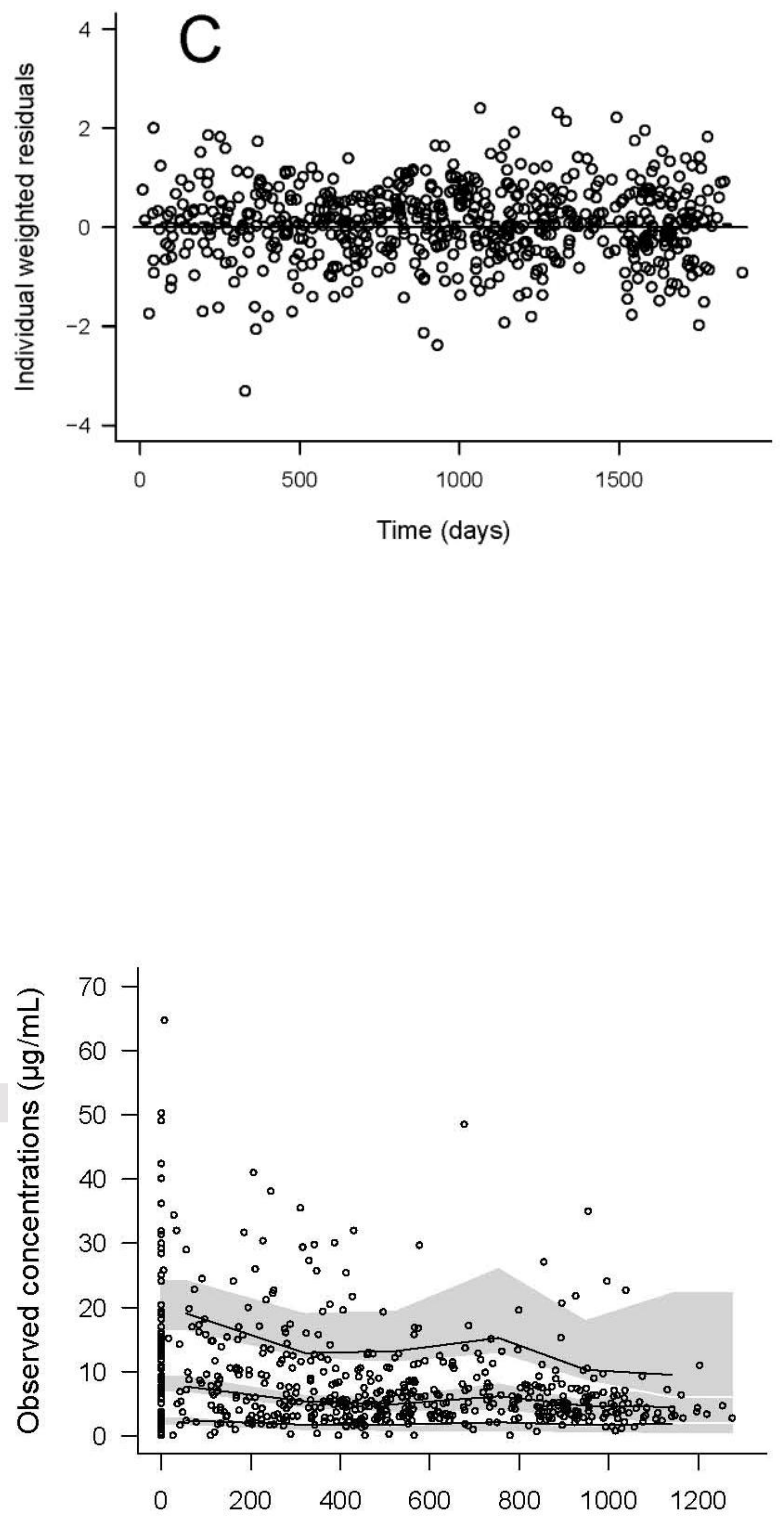

Time since first observation (days)

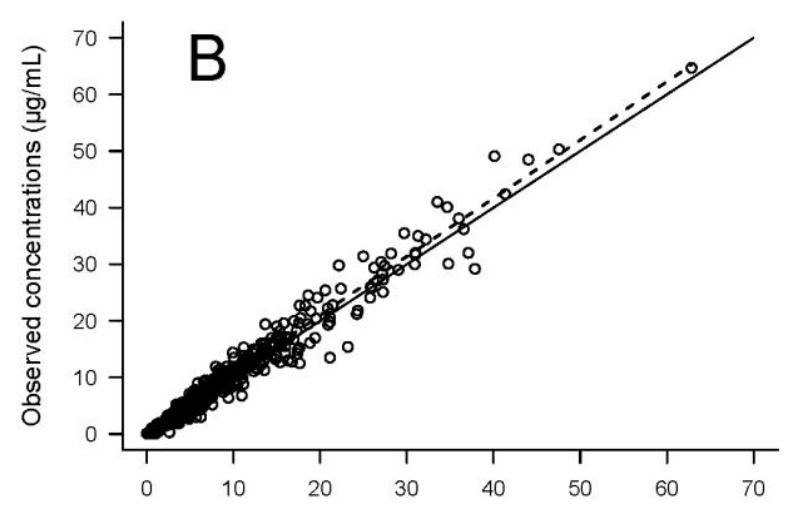

Individual-predicted concentrations $(\mu \mathrm{g} / \mathrm{mL})$
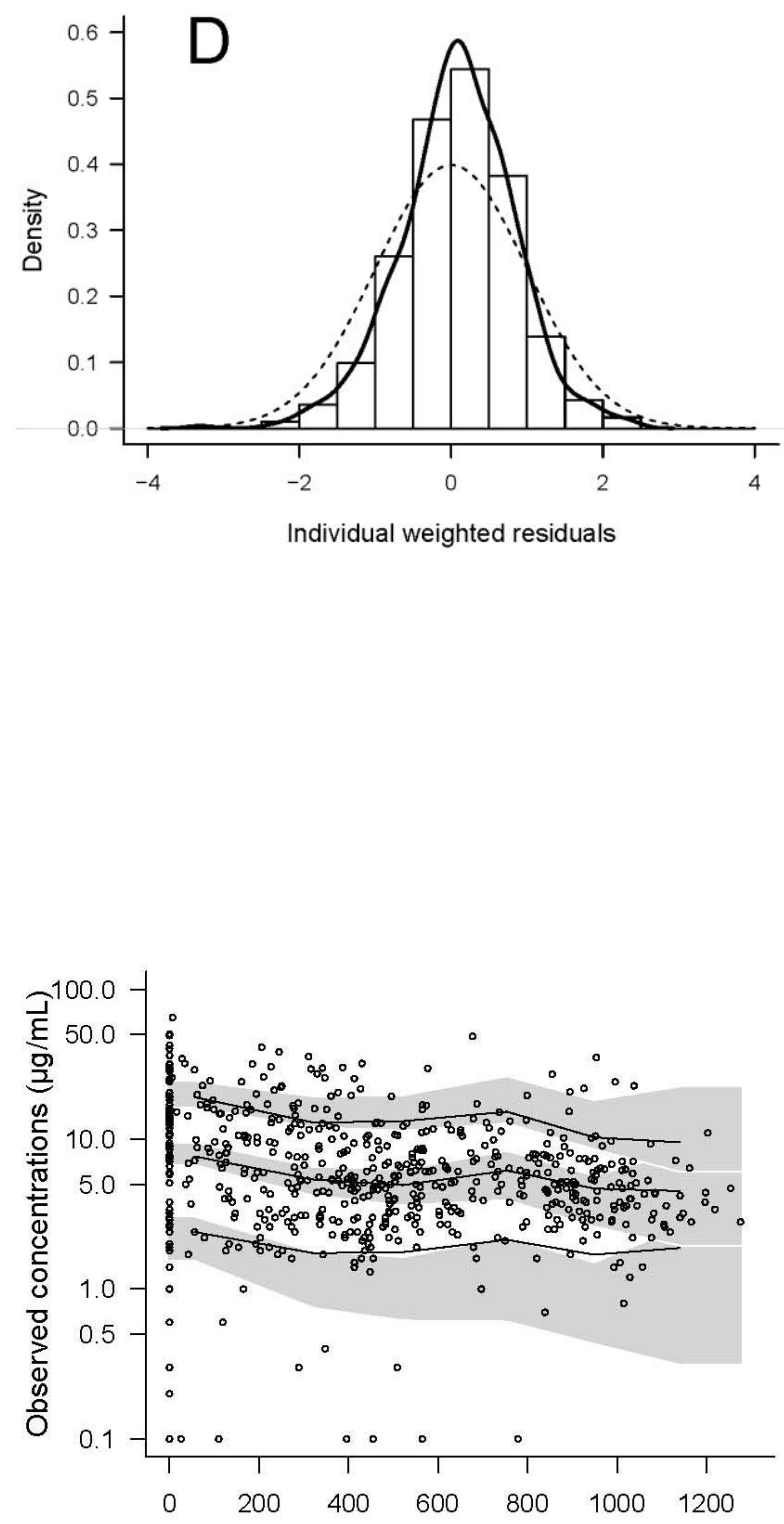

Time since first observation (days) 

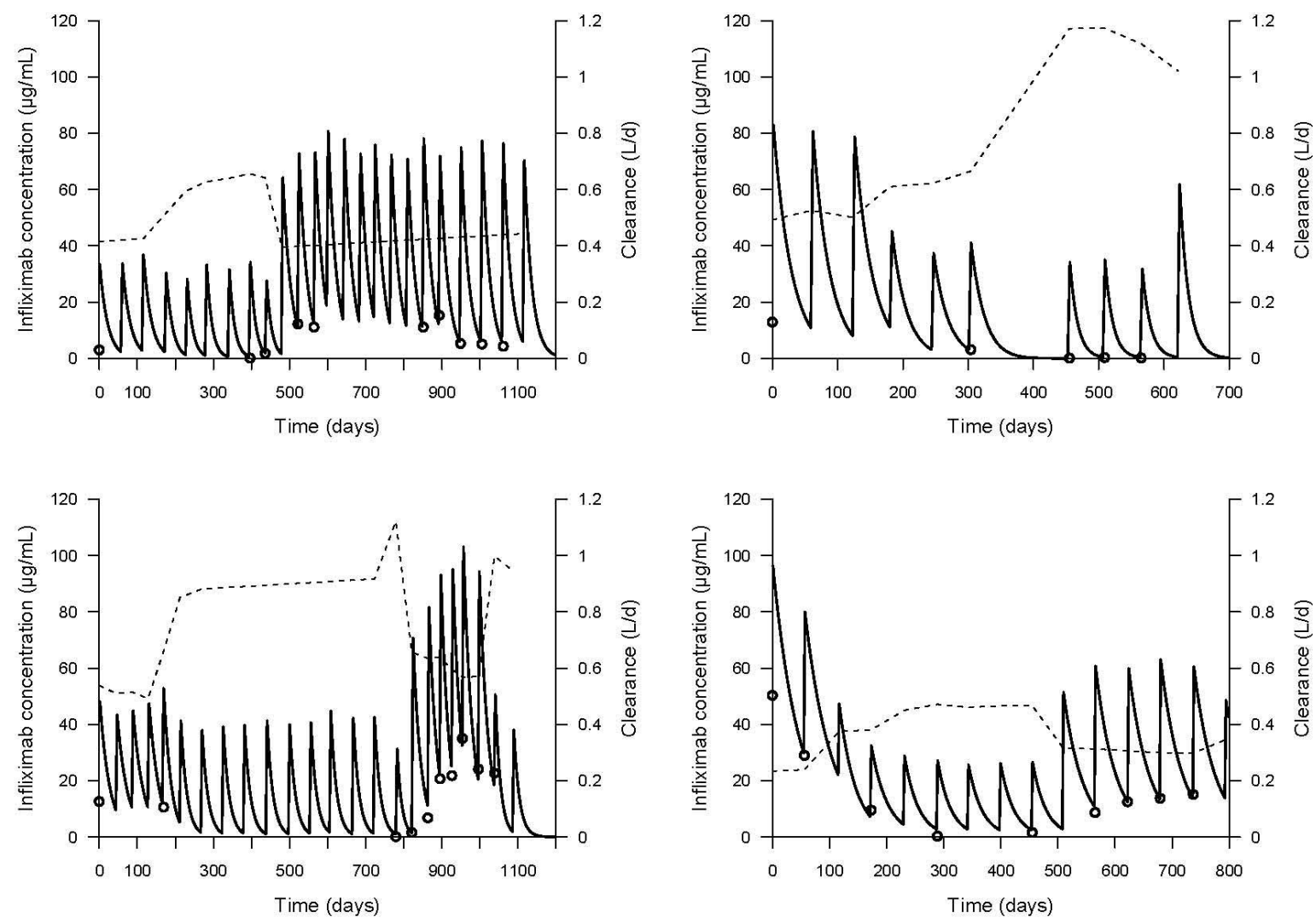

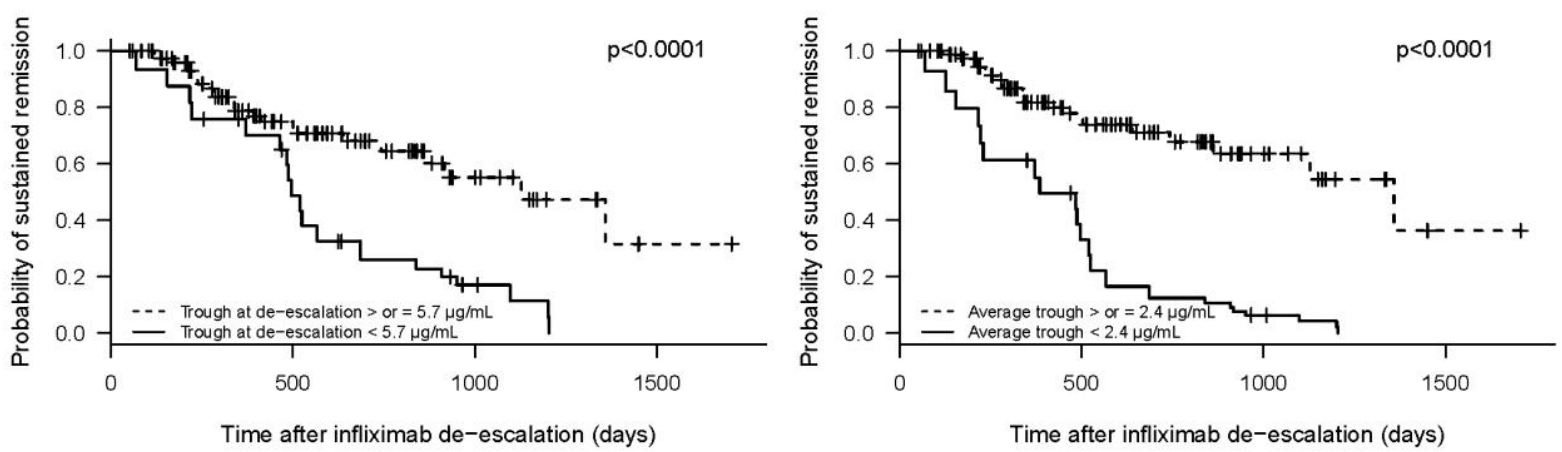

Numbers at risk

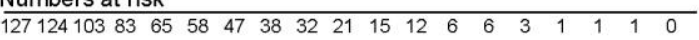
Numbers at risk

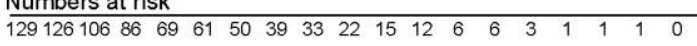
$\begin{array}{lllllllllllllllllll}28 & 27 & 26 & 23 & 21 & 16 & 13 & 10 & 10 & 9 & 4 & 2 & 2 & 0 & 0 & 0 & 0 & 0 & 0\end{array}$
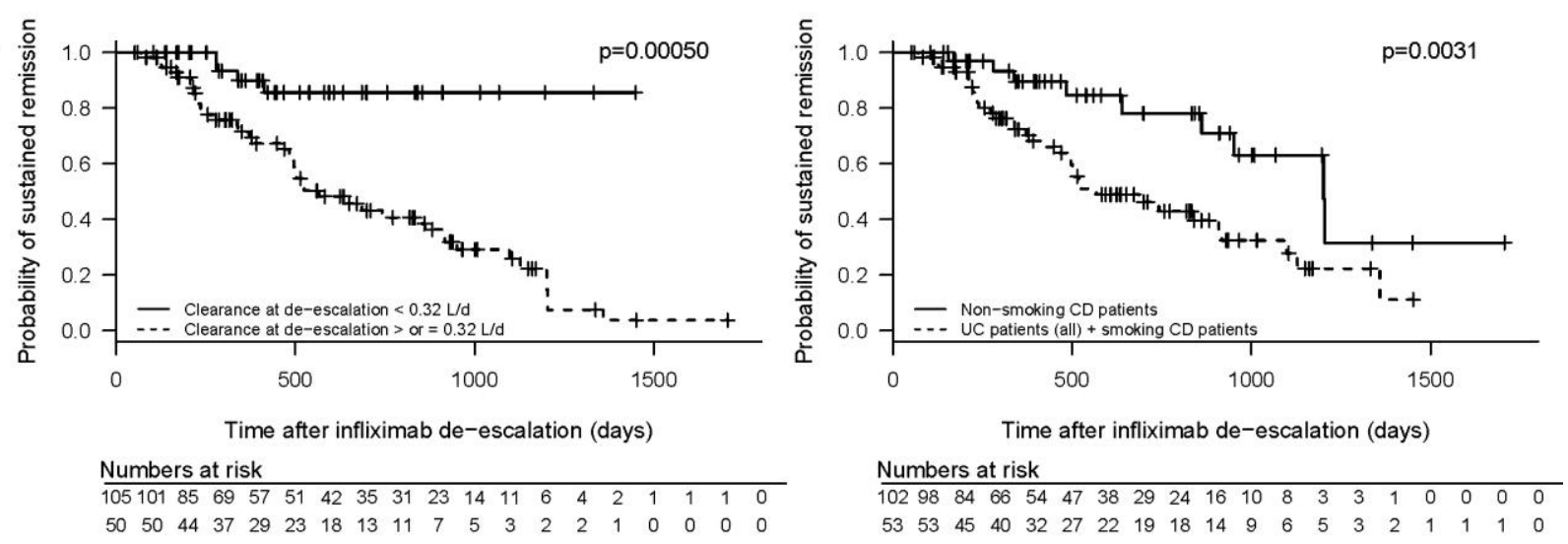